\title{
Graded Manifold Theory as the Geometry of Supersymmetry ${ }^{\star}$
}

\author{
John Dell ${ }^{1}$ and Lee Smolin ${ }^{2}$ \\ 1 Department of Physics and Astronomy, University of Maryland, \\ College Park, Maryland 20742, USA \\ 2 Lyman Laboratory of Physics, Harvard University, Cambridge, Massachusetts 02138, USA
}

\begin{abstract}
Building upon Kostant's graded manifold theory, we present a new way of introducing spinors into the spacetime manifold, by expanding the algebra of functions on spacetime to a graded algebra. The elements of differential geometry are generalized to accomodate the expanded algebra of functions and in this enriched geometry we find the elements of supersymmetry and of supergravity theory. The geometrical role of the supergravity fields is discussed and a derivation of their transformation rules is given.
\end{abstract}

\section{Introduction}

Symmetry transformations mixing particles of different spin were introduced several years ago under the name of supersymmetry [1,2]. In the simplest case, due to Wess and Zumino [2], a scalar field $\phi$ and an anticommuting spinor field $\chi$ are given the following variations

$$
\begin{aligned}
\delta \phi & =\bar{\varepsilon} \chi \\
\delta \chi & =(\not \partial \phi) \varepsilon,
\end{aligned}
$$

where $\varepsilon$ is taken to be a constant anticommuting spinor. The action integral for a free scalar field and a free spinor field is easily seen to be invariant under these variations [2].

A characteristic feature of supersymmetry transformations is that the symmetrized product of two supersymmetry transformations is always an infinitesimal translation. One finds that,

$$
\left(\delta_{1} \delta_{2}+\delta_{2} \delta_{1}\right) \phi, \chi=2 \bar{\varepsilon}_{1} \gamma^{\mu} \varepsilon_{2} \partial_{\mu} \phi, \chi .
$$

Thus supersymmetry transformations are in some sense an extension of the algebra of infinitesimal spacetime translations. This suggested that a theory of local supersymmetry, where the parameters $\varepsilon$ are taken to be functions of

* Research supported in part by the National Science Foundation under Grant Nos. PHY77-22864 and PHY77-05299 
spacetime, should have something to do with gravitation, because a theory invariant under local supersymmetry transformations would by closure also have to be invariant under local infinitesimal translations, and these latter play a role in the general theory of relativity. Indeed an action for general relativity coupled to an anticommuting spin $3 / 2$ field $\psi_{\mu}$ was found to be invariant under the following local supersymmetry transformations $[3,4]$

$$
\begin{aligned}
\delta g_{\mu \nu} & =\bar{\varepsilon} \gamma_{(\mu} \psi_{\nu)}, \\
\delta \psi_{\mu} & =\nabla_{\mu} \varepsilon .
\end{aligned}
$$

Taking again the antisymmetric product one easily sees that

$$
\left(\delta_{1} \delta_{2}+\delta_{2} \delta_{1}\right) g_{\mu v}=\nabla_{(\mu} \xi_{v)},
$$

where

$$
\xi_{v}=2 \bar{\varepsilon}_{1} \gamma_{v} \varepsilon_{2} .
$$

Recalling that Eq. (1.4) represents the Lie derivative of the metric along a vector field given by Eq. (1.5) [5], we see that local supersymmetry transformations should constitute, in some sense, an extension of the algebra of vector fields on spacetime.

This theory of gravity coupled to a spin $3 / 2$ particle, called supergravity, has seen an enormous amount of development in the past few years. Supersymmetry may very well be an ingredient in the eventual solution of two of the oldest and most difficult problems in theoretical physics, that of understanding the relationship between gravitation and the other forces in nature, and that of building a consistent quantum theory of gravity. However in spite of this wonderful progress the conceptual and mathematical foundations of supersymmetry are not well understood. The idea of supersymmetry and the motivations given for it involve an extension of our ideas about the structure of spacetime and the relation of spacetime structure to the elementary particles that move in it. However, exactly what these ideas are, and what they imply for physics, has never been clear. We hope to clarify the nature of supersymmetry theories by developing a mathematical framework for supergravity that is a direct extension of the mathematics that we use to describe spacetime. For this purpose we have found Kostant's notion of a graded manifold [6] to be a useful generalization of differential geometry, and we have taken his work as our starting point.

The basic idea is to extend the algebra of functions on the spacetime manifold. These functions represent physically the classical observables of a scalar field, and we extend the algebra of observables to include the observables of fields with spin. Very roughly, a differential manifold on which the algebra of functions has been extended to include anticommuting as well as commuting elements is called a graded manifold. In differential geometry all of the intrinsic structures on the manifold are defined ultimately in terms of the algebra of functions, and it turns out to be straightforward to generalize these structures to accommodate the expanded algebra of functions on a graded manifold. We then discover a representation of the elements of supersymmetry theory in the geometrical structure of the graded manifold, and we thus discover in what sense supersymmetry transformations are, and are not, a generalization of spacetime trans- 
lations. We also discover a geometrical interpretation for the spin $3 / 2$ field, and we find a derivation of the supergravity transformation laws, Eqs. (1.3a) and (1.3b).

Before going on we would like to discuss in more detail what questions are left unanswered by the standard formulations of supersymmetry and how the formulation presented here may clear them up.

The first problem concerns the nature of the supersymmetry transformations themselves. In physics there are two very different kinds of symmetry transformations, called passive and active transformations. Passive transformations are transformations between different mathematical descriptions of the same physical situation; for example, coordinate transformations and gauge transformations are of this type. Passive transformations correspond to different ways of describing the same physical objects, and it follows that well defined geometric objects are invariant under passive transformations.

Active transformations, on the other hand, correspond to changes in some aspect of the actual physical system. One example is isospin transformations where protons are substituted for neutrons in an experiment, another is spacetime translations, where objects are actually moved from one place to another on the spacetime manifold. Local geometric objects are certainly not, in general, invariant under active transformations but must transform to reflect the active transformation of the physical situation they correspond to. However, in particular circumstances an active transformation may leave the physics of the situation unchanged and when this is true, and the transformation is a continuous operation, Noether's theorem tells us that conserved currents must exist [7]. In the absence of gravitational fields spacetime is homogeneous, and the physics of any system is unchanged if it is moved from one part of spacetime to another. The result, by Noether's theorem is of course the conservation of energy and momentum. In general spacetime is not homogeneous, and the physical behavior of systems is altered if they are moved from one place to another. As a consequence of this, energy and momentum are no longer in general conserved.

We saw in Eqs. (1.4) and (1.5) that the symmetrical product of supersymmetry transformations corresponds to Lie derivation by a vector field. The Lie derivative is defined in terms of an active transformation in which geometric objects are dragged infinitesimally along the flow lines of a vector field, and it follows from this that supersymmetry transformations must also be some kind of active transformations. For the product of two passive transformations, each leaving geometrical objects invariant must leave geometric objects invariant and hence must be also a passive transformation. In terms of graded manifolds we shall see that the set of vector fields on the manifold is expanded and that Lie derivation by some of the new, odd, vector fields generate local supersymmetry transformations. Moreover the relationship between supersymmetry and spacetime transformations follows naturally from the Lie algebra of our expanded vector fields.

The realization that local supersymmetry transformations are active transformations has important consequences for the theory of supersymmetry. It means that there do not exist any local objects that are invariant under supersymmetry transformations, in particular there cannot be any manifestly supersymmetry invariant local Lagrangian. The fact that supersymmetry transformations are active transformations means that the situation is rather different from that of 
gauge invariance in Yang-Mills theory, and any manifestly invariant objects must be global objects, defined as integrals over the whole manifold. This result should not be surprising as it is the case in all known supersymmetry theories that it is the action and not the Lagrangian that is invariant.

The active character of supersymmetry transformations has consequences for another important question from supergravity theory, what exactly is the geometric role of the spin $3 / 2$ particle $\psi_{\mu}$ ? It has been assumed by many people that the spin $3 / 2$ field is a connection field that plays the same role with respect to supersymmetry invariance that the electromagnetic field plays with respect to its gauge invariance. However, if supersymmetry transformations are active rather than passive transformations then, by analogy with local translations, we should not expect to construct a covariant derivative for supersymmetry.

But if the $\psi_{\mu}$ field is not a connection then how does one interpret its transformation law, Eq. (1.3b), which resembles the transformation law for a gauge field under a gauge transformation? In the geometry of graded manifolds we will find that the metric of spacetime is extended following the extension of the algebra of functions, and it will be a natural hypothesis to associate the spin $3 / 2$ field with one of the new pieces of the metric. We will then see that Eq. (1.3b) can be understood as the result of Lie differentiation of the odd part of the metric by an odd vector field, and thus is analogous to the Lie differentiation of the spacetime metric by a normal vector field as in Eq. (1.4).

We find that we are also able to answer some of the questions concerning the supersymmetry transformation law for the metric, Eq. (1.3a). An interpretation of supersymmetry as a passive symmetry transformations implies that the geometry of spacetime is not well defined as the metric structure would depend on the choice of gauge. In particular as the metric structure defines the light cones, and hence the casual structure on spacetime, it is not at all clear how to define classical or quantum field theory in this situation. Moreover the metric structure is directly measurable in terms of rulers and clocks whereas it is not the case in a normal gauge theory that the variables that transform under gauge transformations are in any way observable. Thus a proponent of a passive interpretation of supersymmetry would have to not only provide us with a mechanism for fixing the gauge but would have to provide us with a way to make sense of physics in the situation that the gauge is not fixed. Moreover he or she would have to explain why we could not in that situation use rulers or clocks, or light signals, or the motion of goodesics to establish the spacetime geometry.

In general, as none of the normally covariant geometric objects, such as the curvature or tortion tensors are now meaningfully defined, the acceptance of a passive interpretation of supersymmetry necessitates a truly radical revision of our ideas about spacetime, and we know of no positive steps in this direction. It is thus very gratifying to find that we have to regard supersymmetry transformations as active transformations, as in this case we have none of these problems. We will find that we are able to derive and interpret Eq. (1.3a) in terms of the mixing of the various components of the contravariant metric under the action of an odd vector field.

In Sect. II we describe Kostant's formulation of graded manifold theory, and a very useful representation of graded manifolds due to Batchelor. Then, in view of 
their intended use, we impose additional structure, defining what we call a spin graded manifold. In Sect. III we show how supersymmetry transformations are found in the tangent space of a spin-graded manifold, and we make some comparisons between superspace and graded manifold theory. In Sect. IV we see that local supersymmetry transformations are generated by odd vector fields, and we show how, if we consider the spin $3 / 2$ field to be part of the metric defined on the tangent space of a spin graded manifold, we can derive the supergravity transformation laws. In Sect. V we summarize our results and argue that graded manifold theory provides a geometric formulation for supersymmetry that is more faithful to the physical content of the theory than are the usual superspace constructions.

\section{Graded Manifold Theory}

The papers of Kostant [6] and of Batchelor [8] on graded manifold theory are written in the language of sheaves and bundles and thus are not easily accessible to most physicists. We have tried to write this section so that it can be understood by anyone who is familiar with differential geometry as it is developed in the standard treatments of general relativity [5]. What we do is give a description of those elements of graded manifold theory relevant to our purposes here, thus our exposition consists almost entirely of motivation and definitions. A few theorems are stated without proof, many are simple generalizations of theorems in ordinary differential geometry. For the rest, and for more details, we refer the reader to the original papers $[6,8]$.

Consider the set of $C^{\infty}$ functions defined over an open set $U$ of a manifold $M$. This set is closed under the usual addition and multiplication of functions and thus forms an algebra (actually a commutative ring) which we denote by $C^{\infty}(U)$. In classical field theory $C^{\infty}(U)$ is interpreted as the algebra of observables of a neutral scalar field in the region $U$. The basic idea of our construction is to expand this algebra to allow for the description of fields possessing spin. The new algebra, called $A(U)$ is taken to be graded commutative, that is it is the direct sum (in the vector space sense) of two pieces

$$
A(U)=A(U)_{0}+A(U)_{1}
$$

such that if $f, g \in A(U)_{0}$ and $s, t \in A(U)_{1}$ then

$$
f g=g f \in A(U)_{0}, \quad f_{s}=s f \in A(U)_{1}, \quad s t=-t s \in A(U)_{0} .
$$

We call $A(U)_{0}$ the even part and $A(U)_{1}$ the odd part of $A(U)$. An element is called homogeneous if it is purely even or purely odd, by Eq. (2.1) any element is the sum of an even and odd element. If $f \in A(U)_{0}$ we define $o(f)=0$, if $f \in A(U)_{1}$ then $o(f)=1$. We define the graded commutator of two homogeneous elements $f$ and $g$ of $A(U)$ to be

$$
[f, g]=f g-(-1)^{0(f) 0(g)} g f \text {. }
$$

Although it is not usually stressed, the fundamental constructs of differential geometry may be constructed directly from the algebra of $C^{\infty}$ functions. Indeed, 
given the algebra $C^{\infty}(U)$ the points of the set $U$ can be reconstructed as a basis for the dual space to $C^{\infty}(U)$, the dual space being defined as the set of all linear maps from $C^{\infty}(U)$ to the real numbers. The tangent space is defined in terms of operations on $C^{\infty}(U)$ and most of the rest of the structures are defined in terms of the tangent space. We will develop graded differential geometry by following the usual constructions of differential geometry, except that at each step we will generalize the construction by letting the algebra $A(U)$ play the role played by $C^{\infty}(U)$. In this way we will define the graded tangent space, graded forms, metric and connections. However, we must first describe in more detail the properties of a graded manifold.

$A(U)$ may be split into a direct sum of two pieces, one of which contains all of its nilpotent elements. That is

$$
A(U)=A^{0}(U)+A^{1}(U)
$$

where $f \in A^{1}(U)$ implies that there exists a finite $n$ such that $f^{n}=0$. Note that while $A(U)_{1} \subset A^{1}(U)$ and $A^{0}(U) \subset A(U)_{0}$ there are also elements that are even and nilpotent. [In fact $A^{1}(U)$ is the nilpoint ideal generated by $A(U)_{1}$.]

In order that the algebra $A(U)$ be in the simplest way possible an extension of $C^{\infty}(U)$ we shall require that $A^{0}(U)$ be isomorphic to $C^{\infty}(U)$. That is we shall require that there exists a homomorphism

$$
\infty: A(U) \rightarrow C^{\infty}(U)
$$

such that

$$
\infty: A^{0}(U) \rightarrow C^{\infty}(U)
$$

is an isomorphism and

$$
\propto: A^{1}(U) \rightarrow 0 \text {. }
$$

This map will have a natural extension to all of the structures we shall define on a graded manifold such that there will always be an unambiguous projection to the analogous structure defined in terms of $C^{\infty}(U)$.

The algebra $A(U)$ has a very useful decomposition which is as follows. We shall consider odd elements $S^{1}, S^{2}, \ldots, S^{n} \in A(U)_{1}$ to be algebraically independent if $S^{1} S^{2} \ldots S^{n} \neq 0$. For any graded algebra there is a unique $m$ which is the maximal number of algebraically independent odd elements. $m$ is called the odd dimension of the algebra $A(U)$. Given a particular choice of $m$ algebraically independent odd elements $S^{A}, A=1, \ldots, m$, consider the set consisting of the $S^{A}$ 's, their products and the element $1 \in A^{0}(U)$. This set has the structure of an exterior algebra, and is denoted by $D(U)$. One can then show that the algebra $A(U)$ is isomorphic to the product of the algebras $A^{0}(U)$ and $D(U)$, that is that there exists an isomorphism

$$
A^{0}(U) \otimes D(U) \rightarrow A(U)
$$

given by

$$
f \otimes t \rightarrow f t
$$


for $f \in A^{0}(U)$ and $t \in D(U)$. Thus, given a choice of $S^{A}$ 's any element $f \in A(U)$ may be written as

$$
f=f^{0}+f_{A} S^{A}+f_{A B} S^{A} S^{B}+\ldots+f^{m} S^{1} S^{2} \ldots S^{m},
$$

where all of the $f$ 's are $C^{\infty}$ functions and repeated indices are summed over as usual.

The choice of algebraically independent elements is not unique. Given one set of $m$ algebraically independent elements $S^{A}$ any other set of $m$ elements

$$
S^{A^{\prime}}=M_{B}^{A^{\prime}} S^{B}+M_{B C D}^{A^{\prime}} S^{B} S^{C} S^{D}+\ldots
$$

will be algebraically independent as long as $M_{B}^{A^{\prime}}$ is a non-singular matrix with real valued coefficients. A different choice of the $S^{A}$ results in a different exterior algebra $D(U)$ and a different decomposition in Eq. (2.5), leaving in general only $f^{0}$, the $C^{\infty}$ part, fixed. For the applications considered in this paper we will always be interested in decompositions with respect to a fixed choice of the $S^{A}$ 's.

This completes our discussion of the basic properties of the algebra $A(U)$. For our purposes the important result is Eq. (2.5) which may be expressed by saying that $A(U)$ is just the algebra of the $C^{\infty}$ functions on the set $U$ extended by the inclusion of the $m$ odd elements $S^{A}$. What we must now do is explain how the different algebras $A(U)$ defined over the different open sets of the manifold are to fit together.

We do this by generalizing from the case of $C^{\infty}$ functions. Given any two open sets, $U$ and $V$, on $M$ such that $V \subseteq U$ then there exists a natural map

$$
\varrho_{U, V}: C^{\infty}(U) \rightarrow C^{\infty}(V)
$$

defined by restricting the function defined on the region $U$ to the region $V$. This restriction map satisfies the property:

1) If $W \leqq V \subseteq U$, then

$$
\varrho_{U, W}=\varrho_{U, V} \varrho_{V, W}
$$

If, in addition $U=\bigcup_{i \in \Lambda} U_{i}$ is an open covering of the set $U$ than it can be shown that the following properties hold:

2) If $f, g \in C^{\infty}(U)$, then $\varrho_{U, V_{i}}(f)=\varrho_{U, V_{i}}(g) \forall i \in \Lambda$ implies $f=g$.

3) If $h_{i} \in C^{\infty}\left(U_{i}\right)$ is given for all $i \in \Lambda$ such that

$$
\varrho_{V_{i}, V_{i} \cap V_{j}}\left(h_{i}\right)=\varrho_{V_{j}, V_{i} \cap V_{j}}\left(h_{j}\right)
$$

for all $i, j \in \Lambda$ than there exists an element $h \in C^{\infty}(U)$ [unique by property 2)] such that $\varrho_{V, V_{i}}(h)=h_{i}$.

These properties of $C^{\infty}$ functions are abstracted to give the definition of a sheaf:

If we assign to every open set $U$ of a manifold $M$ an algebra $B(U)$ such that for every $U$ and $V$ such that $V \leqq U$ there exists a map

$$
\varrho_{U, V}: B(U) \rightarrow B(V)
$$

satisfying properties 1)-3) than we say we have a sheaf of $B$ algebras over $M$. 
Given the sheaf of $C^{\infty}$ functions we can construct a sheaf of graded algebra $A(U)$ with the properties discussed. In each $A(U)$ we pick out a fixed set of $m$ algebraically independent elements denoted by $S_{U}^{A}$. We then define, for all $V \subseteq U$, the restriction map

$$
\varrho_{U, V}: A(U) \rightarrow A(V)
$$

to be an algebra homomorphism $\left(\varrho_{U, V}(f g)=\varrho_{U, V}(f) \varrho_{U, V}(g)\right)$ such that acting on $C^{\infty}$ functions it gives the normal restriction map for $C^{\infty}$ functions and such that $\varrho_{U, V}\left(S_{U}^{A}\right)=S_{V}^{A}$. One may easily check that the restriction map defined in this way satisfies the properties 1)-3). It is also clear that there are at least as many restriction maps as there are choices of the $S_{U}^{A}$ for each $U$.

We are now ready to give Kostant's definition of a graded manifold [6]:

An $n$ dimensional differentiable manifold which is covered by a sheaf of graded commutative algebras $A(U)$ is called a graded manifold if, for all $U \subseteq M$,

1) There exists a homomorphism $\infty: A(U) \rightarrow C^{\infty}(U)$.

2) There exists an exterior algebra $D(U) \subset A(U)$ such that $A(U)$ is isomorphic to the product of $D(U)$ and $C^{\infty}(U)$.

The elements of the algebras $A(U)$ will be called, when no confusion can arise, the elements of the graded manifold. One can show that for a given graded manifold all of the algebras $A(U)$ must have the same odd dimension $m . m$ is then called the odd dimension, and $n$ the even dimension of the graded manifold.

The definition of a graded manifold summarizes our discussion up to this point. A graded manifold is essentially an ordinary manifold whose algebra of functions has been extended to a graded commutative algebra. However, this description of graded manifolds has a certain disadvantage for the application to physics which follows from the fact that the algebras $A(U)$ are assigned to the open sets rather than to the points of the manifold. One cannot evaluate an element of a graded manifold at a point, in the manner of a normal function, without first specifying a particular open set containing that point and then a particular set of $S^{A}$ 's for that set. Thus, the description is in a peculiar sense non-local.

One might then wonder whether it is possible to give an equivalent description of a graded manifold in terms of local objects. Batchelor has answered this question by proving that there exists a local construction that is isomorphic to any given graded manifold [8]. Before proceeding to this local construction we give rough definitions for two terms, vector bundle and section, whose use will simplify the subsequent discussion.

Given a vector space $V$ we may associate a copy of it, $V_{x}$ to each point $x$ of a manifold $M$. The set of all the $V_{x}$ for all $x \in M$ is called a vector bundle $\mathscr{V}$ over $M$ with typical fibre $V$. Since observables in field theory often take their values in some vector space the concept of a vector bundle is a very general one that encompasses the idea of a locally defined physical field. A section through a vector bundle $\mathscr{V}$ over an open set $U \subseteq M$ is a smooth map from $M$ into $\mathscr{V}$ that assigns to each $x \in U$ one element out of $V_{x}$. For example if $V=R$, the real numbers, then the vector bundle is called a line bundle and, given any $U \subseteq M$, the sections through the line bundle over the set $U$ are isomorphic to the space of $C^{\infty}$ real valued functions over that set. 
We now give the local construction of a graded manifold. Given a $m$ dimensional vector space $V$ over a field $R$, we define the exterior algebra of $V$, Ext $V$, to be the direct sum of vector spaces $(\operatorname{Ext} V)_{i}$

$$
\text { Ext } V=\bigoplus_{i=0}^{m}(\operatorname{Ext} V)_{i}
$$

where $(\text { Ext } V)_{i}$ is the $i$-fold antisymmetric tensor product of $V$ with itself, $(\text { Ext } V)_{i} \ni w_{1} \wedge w_{2} \wedge \ldots \wedge w_{i}$ where $w_{k} \in V$ and $w \wedge v=w \otimes v-v \otimes w$. (Ext $\left.V\right)_{0}$ is taken to be the field $R$, and of course $(\text { Ext } V)_{1}=V$. $\wedge$ defines a product structure on Ext $V, a b=a \wedge b$. Note that if $a \in(\operatorname{Ext} V)_{i}$ and $b \in(\operatorname{Ext} V)_{j}, a b=(-1)^{i j} b a$ so that Ext $V$ is a graded vector space.

If $b^{A}, A=1, \ldots, m$ are a set of basic elements for $V$, then the set consisting of $1 \in R$ and all of the products $b^{A}, b^{A} b^{B}, \ldots, b^{1} b^{2} \ldots b^{m}$ form a set of basic elements for Ext $V$. Hence, any element $r$ Ext $V$ may be expressed as

$$
r=r_{0}+r_{a} b^{A}+r_{a b} b^{A} b^{B}+\ldots r^{m} b^{1} b^{2} \ldots b^{m},
$$

where all of the $r$ 's are elements of $R$.

The resemblance of Eq. (2.6) to Eq. (2.5) is a result of the fact that the exterior algebra $D(U)$ constructed from a graded algebra with odd dimension $m$ is isomorphic to the algebra of basic elements of Ext $V$ for any $m$ dimensional vector space $V$. We proceed to construct a graded manifold by making a vector bundle over an $n$ dimensional manifold whose typical fiber is Ext $V$. This is called the exterior bundle associated with the vector bundle $\mathscr{V}$ and is denoted Ext $\mathscr{V}$. Clearly, Ext $\mathscr{V}$ is the direct sum of $m+1$ vector bundles,

$$
\text { Ext } \mathscr{V}=\bigoplus_{i=0}^{m}(\operatorname{Ext} \mathscr{V})_{i}
$$

Again, $(\text { Ext } \mathscr{V})_{1}=\mathscr{V}$ and $(\text { Ext } \mathscr{V})_{0}=\mathscr{R}$, a line trivial bundle with typical fibre $R$. We take $R$ to be the real numbers, than the sections of $\mathscr{R}$ over a set $U$ are isomorphic to $C^{\infty}(U)$.

Over an open set $U$ we may pick a field of basic elements $b^{A}$ for $\mathscr{V}$, then it is easy to see that any smooth section through Ext $\mathscr{V}$ must have the following form

$$
f=f^{0}+f_{A} b^{A}+f_{A B} b^{A} b^{B}+\ldots+f^{m} b^{1} b^{2} \ldots b^{m},
$$

where the $f$ 's are elements of $C^{\infty}(U)$ as in Eq. (2.5); however, in this case the $b^{A}$ 's are associated with points rather than open sets. The sections $f$ may thus be thought of as functions over $U$ taking values in the exterior algebra Ext $V$.

Clearly, Ext $\mathscr{V}$ is a graded manifold. Batchelor's theorem states that the two constructions, one in terms of graded commutative algebras, the other in terms of exterior bundles, are completely equivalent. The precise statement of the theorem is: If $\mathscr{V}$ is a real vector bundle over a smooth manifold $M$, let Ext $\mathscr{V}$ be the associated exterior bundles and let $\Gamma(\operatorname{Ext} \mathscr{V})$ be the sheaf of sections of Ext $\mathscr{V}$. Then, every graded manifold over $M$ is isomorphic to $\Gamma($ Ext $\mathscr{V})$ for some vector bundle $\mathscr{V}$ over $M[8]$. it here.

The proof of the theorem involves sheaf cohomology, and we shall not discuss 
We would now like to use the elements of a graded manifold to describe a multiplet of physical fields on spacetime. In order to do this, we must define the action of the local Lorentz group on Ext $\mathscr{V}$. To accomplish this, we shall require that the vector space $V$ transform according to the spinor representation of the Lorentz group. Thus the elements of our graded manifold shall be sections through the exterior bundle associated with the spin bundle of our spacetime manifold $M$. We propose to call this structure a spin-graded manifold, and it will be the basis for all of our constructions in the rest of the paper.

The $S^{A}$ will from now on be considered to be basis fields for the spin bundle $\mathscr{V}$, and any section through $\mathscr{V}$ over an open set $U$ can be written as $f=f_{A} S^{A}$ where $f_{A} \in C^{\infty}(U)$. Thus our spinors have real valued coefficient functions rather than Grassman valued, as is usual in formulations involving supersymmetry. However, this formulation, in which classical spinor fields are treated as anticommuting Grassman algebras is only really appropriate for quantum field theory, to implement the spin-statistics relation in the functional integral. For a purely classical formulation, such as we are concerned with in this paper, real valued spinors are the appropriate thing, otherwise one is led to writing nonsensical equations in which one side is nilpotent, and the other is $C^{\infty}$. Also, note that since the coefficients are real valued functions, our spinors are Majorana.

The spin bundle has a metric structure given by the charge conjugation matrix $C^{A B}, C_{A B}$, which is used to raise and lower spinor indices

$$
\begin{aligned}
& S_{A}=C_{A B} S^{B}, \\
& S^{A}=C^{A B} S_{B} .
\end{aligned}
$$

The charge conjugation matrix satisfies

$$
C_{A B} C^{B D}=\delta_{A}^{D}
$$

and, as a result of the fact that the spin basis elements anticommute, must be antisymmetric.

In addition, we assume that the spin frames are tied to the usual spacetime frame fields by a bilinear mapping

$$
\gamma: \mathscr{V} \otimes \mathscr{V} \rightarrow \mathscr{T}
$$

where $\mathscr{T}$ is the tangent bundle to $M$. If $e_{a}$ are an orthonormal basis for $\mathscr{T}$ (the veirbeins) we have

$$
\gamma\left(S_{A}, S^{B}\right)=\left(\gamma^{a}\right)_{A}^{B} e_{a}
$$

where the $\left(\gamma^{a}\right)_{A}^{B}$ are the usual gamma matrices (in a real representation). We shall take the coefficients $C^{A B}$ and $\left(\gamma^{a}\right)_{A}^{B}$ to be constants; one can show that no additional degrees of freedom are introduced by letting the $\left(\gamma_{a}\right)_{A}^{B}$ be functions of spacetime.

We can thus use the $\gamma$ matrices to write an arbitrary element of a spin graded manifold in terms of quantities that transform irreducibly under local Lorentz rotations.

$$
\begin{aligned}
f= & f_{0}+f_{A} S^{A}+R S_{A} S^{A}+P S_{A}\left(\gamma^{5}\right)_{B}^{A} S^{B} \\
& +V_{a} S_{A}\left(\gamma^{5} \gamma^{a}\right)_{B}^{A} S^{B}+f_{2 A} S^{A} S_{B} S^{B}+f_{2}\left(S_{A} S^{A}\right)^{2} .
\end{aligned}
$$


Thus we see that the elements of a spin graded manifold consist of three scalar fields, one pseudo scalar field, two spinor fields and an axial vector field.

In deriving Eq. (2.1) from Eq. (2.5) we used the well-known symmetry properties of the $\gamma$ matrices [2]; as well as the useful identity

$$
\varepsilon^{A B C D}=C^{A B} C^{C D}-C^{A C} C^{B D}+C^{A D} C^{B C} .
$$

In order to compare formulas like Eq. (2.13) with the usual formulation with suppressed spinor indices (but explicit charge conjugation) it is helpful to remember that the $\gamma$ matrices are defined with one index up and one index down, and all other objects are defined with a raised index. Thus $f_{A}=C_{A B} f^{B}$ is equivalent to $\bar{f}$ and $f_{A} S^{A}$ to $\bar{f}_{S}$.

We now come to the definition of the extended tangent space associated with a graded manifold. We proceed by recalling the definition of the tangent space for an ordinary manifold. The tangent space at a point $p$ of a manifold $M$ is defined to be the set of all linear maps $W_{p}$ from the space of functions to the real numbers satisfying the Liebnitz property.

$$
W_{p}(f g)=W_{p}(f) g(p)+f(p) W_{p}(g)
$$

where $f, g \in C^{\infty}(U)$ and $U$ contains $p$. We see that the definition of the tangent space involves the function algebra and thus we can define a generalized tangent space with $A(U)$ taking the place of $C^{\infty}(U)$. Since the elements of a graded manifold may be thought of as functions taking values in the exterior algebra Ext $V$ [whose general element may be written as Eq. (2.6)], we shall consider the linear maps from $A(U)$ to Ext $V$ that satisfy a graded Leibnitz property. The space of linear maps between two graded vector spaces is graded, even maps are defined as those which preserve the grading while odd maps are those that map between the even and odd subspaces. Thus we make the following definition: The tangent space $T_{p}$ to a point $p$ of a graded manifold $M$ is a graded vector space,

$$
T_{p}=\left(T_{p}\right)_{0}+\left(T_{p}\right)_{1}
$$

consisting of all the linear maps

$$
W_{p}: A(U) \rightarrow \operatorname{Ext} V
$$

such that each homogeneous element satisfies,

$$
W_{p}(f g)=W_{p}(f) g(p)+(-1)^{0(f) 0\left(W_{p}\right)} f(p) W_{p}(g),
$$

where $f, g \in A(U)$ and $p \in U$.

Given a set of coordinates $x^{\mu}$ on $U$ a basis for the ordinary tangent space is given by the partial derivatives $\frac{\partial}{\partial x^{\mu}}$ evaluated at $p$. In the case of graded manifolds one can show [6] that, given coordinates $x^{\mu}$ and a basis $S^{A}$ for $V$ any element of $T_{p}$ can be written as,

$$
W=W^{\mu} \frac{\partial}{\partial x^{\mu}}+W^{A} \frac{\partial}{\partial S^{A}},
$$


where $W^{\mu}$ and $W^{A}$ are elements of the algebra Ext $V \cdot \frac{\partial}{\partial S^{A}}$ is defined by

$$
\frac{\partial}{\partial S^{A}} S^{B}=\delta_{A}^{B}
$$

and the following anticommutation relations

$$
\begin{gathered}
\frac{\partial}{\partial S^{A}} \frac{\partial}{\partial S^{B}}+\frac{\partial}{\partial S^{B}} \frac{\partial}{\partial S^{A}}=\left[\frac{\partial}{\partial S^{A}} \frac{\partial}{\partial S^{B}}\right]=0 \\
\frac{\partial}{\partial S^{A}} \frac{\partial}{\partial x^{\mu}}-\frac{\partial}{\partial x^{\mu}} \frac{\partial}{\partial S^{A}}=\left[\frac{\partial}{\partial S^{A}} \frac{\partial}{\partial x^{\mu}}\right]=0
\end{gathered}
$$

where we have made use of the definition of the graded commutator, Eq. (2.3). In addition, the following equations hold,

$$
\begin{aligned}
& \frac{\partial S^{A}}{\partial x^{\mu}}=0 \\
& \frac{\partial x^{\mu}}{\partial S^{A}}=0 .
\end{aligned}
$$

In the rest of the paper we shall make use of the abbreviations,

$$
\partial_{\mu}=\frac{\partial}{\partial x^{\mu}} \quad \partial_{A}=\frac{\partial}{\partial S^{A}}
$$

It follows from Eq. (2.18) that the graded tangent space is the direct sum of a part that is isomorphic to the normal tangent space and a nilpotent part.

Having defined the tangent space for a graded manifold we proceed to define graded vector fields. Ordinary vector fields are defined to be a smooth map from each point in the manifold to an element of the tangent space over that point, that is as sections through the tangent bundle. We thus define a graded vector field to be a section through the tangent space of a graded manifold. Any graded vector field can be decomposed into a sum of one that is purely even and one that is purely odd; it also can be decomposed by the $\infty$ map into the sum of an ordinary vector field and a nilpotent one. From now on, unless otherwise stated we shall use the term vector field to refer to the general, graded case. The vector fields on a region $U$ of a manifold $M$ can also be thought of as the set of linear maps from the function algebra on $U$ to itself which satisfy the Liebnitz rule. It follows from Eq. (2.18) that the odd vector fields (which must carry an odd number of $S^{A}$ 's and $\partial_{A}$ 's) acting on an element of $A(U)$ map the different coefficient functions, representing fields of different spin into each other. Thus the odd vector fields on a graded manifold may be thought of as infinitesimal supersymmetry transformations. We shall show in the next two sections how the properties of supersymmetry transformations can be understood in terms of this fact.

In order to complete our description of the differential structure of graded manifolds, we need to introduce the cotangent space and differential forms. Given the tangent space $T_{p}$ at a point $p \in M$, we define the cotangent space $T_{p}^{*}$ to be all the linear maps from $T_{p}$ into Ext $V$. 
If $W \in T_{p}$ and $\eta \in T_{p}^{*}$, then the map

$\eta: W \rightarrow \operatorname{Ext} V$

is denoted as usual by $\langle\eta, W\rangle$. As with the tangent space, the cotangent space has an even and an odd part, and a $C^{\infty}$ part and a nilpotent part.

The smooth sections through the cotangent bundle are called, as usual, the 1 -forms of the graded manifold. If $\Omega^{1}(A, U)$ denotes the set of 1 -forms over an open set $U$ of a graded manifold, then there exists a map

$$
d: A(U) \rightarrow \Omega^{1}(A, U)
$$

given by

$$
\langle d f, W\rangle=W(f),
$$

where $f \in A(U)$ and $W$ is a vector field. It follows that a basis for $T_{p}^{*}$ is given by $d x^{\mu}$, $d S^{A}$ where

$$
\begin{aligned}
& \left\langle d x^{\mu}, \partial_{\lambda}\right\rangle=\delta_{\lambda}^{\mu}, \\
& \left\langle d S^{A}, \partial_{B}\right\rangle=\delta_{B}^{A}, \\
& \left\langle d x^{\mu}, \partial_{A}\right\rangle=\left\langle d S^{A}, \partial_{\mu}\right\rangle=0,
\end{aligned}
$$

and that any $\omega \in \Omega^{1}(A, U)$ can be written as

$$
\omega=\omega_{\mu} d x^{\mu}+\omega_{A} d s^{A},
$$

where $\omega_{\mu}, \omega_{A} \in A(U)$. Moreover, we have,

$$
d f=\frac{\partial f}{d x^{\mu}} d x^{\mu}+\frac{\partial f}{\partial S^{A}} d S^{A} .
$$

If $\eta, \omega \in \Omega^{1}(A, U)$, we define the graded exterior product as

$$
\eta \wedge \omega=\eta \otimes \omega-(-1)^{0(\eta) 0(\omega)} \omega \otimes \eta .
$$

We then define the general $(l, m)$ form on a graded manifold to be

$$
\omega=\omega_{\mu_{1} \ldots \mu_{l}, A_{1} \ldots A_{m}} d x^{\mu_{1}} \wedge \ldots \wedge d x^{\mu_{l}} \wedge d S^{A_{1}} \wedge \ldots \wedge d S^{A_{m}} \in \Omega^{l, m}(U, A),
$$

where $\omega_{\mu_{1} \ldots \mu_{l}, A_{1} \ldots A_{m}} \in A(U)$.

We define also

$$
\Omega^{n}(A, U)=\sum_{n=l+m} \otimes \Omega^{l, m}(U, A)
$$

for $n>1$ and

$$
\Omega^{0}(A, U)=A(U)
$$

Cartan's exterior derivative generalizes naturally to this context [6]. If $\omega \in \Omega^{l, m}(A, U)$, then $d \omega \in \Omega^{l+m+1}(A, U)$ is defined by

$$
\begin{aligned}
d \omega= & \frac{\partial \omega_{\mu_{1} \ldots \mu_{l}, A_{1} \ldots A_{m}}}{\partial x^{\lambda}} d x^{\lambda} \wedge d x^{\mu_{1}} \wedge \ldots \wedge d x^{\mu_{l}} \wedge d S^{A_{1}} \wedge \ldots \wedge d S^{A_{m}} \\
& +\frac{\partial \omega_{\mu_{1} \ldots A_{m}}}{\partial S^{D}} d S^{D} \wedge d x^{\mu_{1}} \wedge \ldots \wedge d S^{A_{m}}
\end{aligned}
$$


The reader may easily show that $d^{2}=0$ and that Eq. (2.25) is satisfied.

Finally, we define a particularly useful restriction map on the space of graded tensor fields,

$$
\sim: T_{p} \rightarrow \tilde{T}_{p} .
$$

$\tilde{T}_{p}$ is defined to consist of all the elements of $T_{p}$ which are of the form, $v=v^{\mu} \partial_{\mu}+v^{A} \partial_{A}$,

where $v^{\mu}, v^{A} \in R$. The $\sim$ projection can be easily generalized to arbitrary tensor fields.

\section{Global Supersymmetry}

We have seen how the algebra $A(U)$ contains a multiplet of fields with different spins and parities. In this section we would like to show how global supersymmetry transformations for this multiplet are defined in terms of the constant odd vector fields on $U$. We shall see how the algebra of supersymmetry follows from the graded Lie algebra of vector fields on a graded manifold. Because the notion of a constant vector field is in general only well defined in flat spacetime, we shall consider in this section that we are working in flat spacetime. In the next section we shall discuss local supersymmetry by generalizing to the case of general odd vector fields and curved spacetime.

The formulation of global supersymmetry we outline here completely parallels the usual superspace formulation of global supersymmetry $[9,10]$. At the end of this section we shall compare superspace with graded manifold theory.

Recall that an element $f \in A(U)$ has the general form of Eq. (2.13).

We consider the constant vector field $\varepsilon^{A} \partial_{A}$, where the constant coefficients $\varepsilon^{A}$ are four ordinary numbers, acting on $f$. It can be considered as a map between coefficient functions, in which case it maps the coefficient functions to those with one less $S^{A}$. In particular if we define

$$
\delta_{\varepsilon}^{-} f=\varepsilon^{A} \partial_{A} f
$$

then we find

$$
\begin{aligned}
\delta_{\varepsilon}^{-} f_{0} & =\varepsilon^{A} f_{A}, \\
\delta_{\varepsilon}^{-} f_{A} & =R \varepsilon_{A}+P \varepsilon_{B}\left(\gamma^{5}\right)_{A}^{B}+V_{\alpha} \varepsilon_{B}\left(\gamma^{5} \gamma^{\alpha}\right)_{A}^{B}, \\
\delta_{\varepsilon}^{-} R & =\frac{3}{2} \varepsilon^{A} f_{2 A}, \\
\delta_{\varepsilon}^{-} P & =-f_{A}\left(\gamma^{5}\right)_{B}^{A} \varepsilon^{B}, \\
\delta_{\varepsilon}^{-} A_{\bar{\alpha}} & =f_{A}\left(\gamma^{5} \gamma_{\alpha}\right)_{B}^{A} \varepsilon^{B}, \\
\delta_{\varepsilon}^{-} f_{A}^{2} & =4 f^{2} \varepsilon_{A} .
\end{aligned}
$$

We can compare Eq. (3.2a) with Eq. (1.1a). They express the same transformation with the important difference that usually $\varepsilon$ and $\chi$ are considered to be odd elements of a Grasman algebra whereas here both $f_{A}$ and $\varepsilon^{A}$ are commuting numbers. We have already explained in Sect. II why the coefficient functions are 
ordinary functions, since we wish to interpret supersymmetry in terms of transformations between coefficient functions the $\varepsilon^{A}$ must also be ordinary numbers. Note that in a graded manifold if the $\varepsilon^{A}$ were to be odd, they would have to be of the form

$$
\varepsilon^{A}=\varepsilon_{1}^{A B} S_{B}+\varepsilon_{2}^{A B C D} S_{B} S_{C} S_{D}
$$

in which case would not satisfy any of the properties we would like our supersymmetry generators to have.

While we differ in this respect from the usual formulations of supersymmetry within the context of graded manifolds this choice of $\varepsilon^{A} \in C^{\infty}(U)$ is correct. Moreover our interpretation of supersymmetry transformations in terms of mappings between coefficient functions has one important advantage: we do not have to worry about what it means to say that the variation of an ordinary function is a nilpotent object.

Having defined a lowering supersymmetry operator, we must define a raising operator, that is one that maps coefficient functions up in the number of $S^{A}$. Guided by the idea that the anticommutator of two supersymmetry transformations should be an ordinary derivative we find in $T_{p}$ the object $\varepsilon^{A} \gamma_{A B}^{\lambda} S^{B} \partial_{\lambda}$. The graded commutation relations are

$$
\left[\varepsilon_{1}^{A} \partial_{A}, \varepsilon_{2}^{B} \gamma_{B C}^{\lambda} S^{C} \partial_{\lambda}\right]=\varepsilon_{1}^{A} \gamma_{A B}^{\lambda} \varepsilon_{2}^{B} \partial_{\lambda}
$$

Defining, as before

$$
\delta_{\varepsilon}^{+} f=\varepsilon^{A} \gamma_{A B}^{\lambda} S^{B} \partial_{\lambda} f
$$

we find that,

$$
\begin{aligned}
\delta_{\varepsilon}^{+} f_{A} & =\varepsilon^{C} \gamma_{C A}^{\lambda} \partial_{\lambda} f_{0}, \\
\delta_{\varepsilon}^{+} R & =\frac{1}{4} \varepsilon^{A} \gamma_{A}^{\lambda B} \partial_{\lambda} f_{B}, \\
\delta_{\varepsilon}^{+} P & =-\frac{1}{4} \varepsilon^{A}\left(\gamma^{\lambda} \gamma^{5}\right)_{A}^{B} \partial_{\lambda} f_{B}, \\
\delta_{\varepsilon}^{+} A_{\alpha} & =\varepsilon^{A}\left(\gamma^{\lambda} \gamma^{5} \gamma_{\alpha}\right)_{A}^{B} \partial_{\lambda} f_{B} \\
\delta_{\varepsilon}^{+} f_{A}^{2} & =\frac{3}{2}\left(\varepsilon^{E} \gamma_{E A}^{\lambda} \partial_{\lambda} R+\varepsilon^{E}\left(\gamma^{\lambda} \gamma_{5}\right)_{E A} \partial_{\lambda} P_{5}+\varepsilon^{E}\left(\gamma^{\lambda} \gamma^{5} \gamma^{2}\right)_{E A} \partial_{\lambda} A_{\alpha}\right), \\
\delta^{+} f_{2} & =\frac{1}{4} \varepsilon^{A} \gamma_{A}^{\lambda B} \partial_{\lambda} f_{2 B} .
\end{aligned}
$$

Note again that Eq. (3.5a) gives the correct transformation law for the WessZumino multiplet, Eq. (1.1b). We can form the total supersymmetry operator as the sum of the raising and lowering operator, and we denote its action on $f \in A(U)$ by

$$
\varepsilon(f)=\varepsilon^{A}\left(\partial_{A}+\gamma_{A B}^{\lambda} S^{B} \partial_{\lambda}\right) f .
$$

The notation is chosen to comply with the usual notation for the action of a vector field on a function. The (graded) commutation relationship

$$
\left[\varepsilon_{1}, \varepsilon_{2}\right]=2 \varepsilon_{1}^{A} \gamma_{A B}^{\lambda} \varepsilon_{2}^{B} \partial_{\lambda}
$$

is then seen to be just a piece of the Lie algebra of vector fields.

Note that while all ordinary constant vector fields on spacetime commute, this is not the case for graded vector fields. 
Note that in order that Eq. (3.6) and (3.7) be dimensionally consistent, we must assign dimensions to $S^{A}$ of square root of length, and to $\partial_{A}$ of inverse square root of length. Whether this assignment of dimensions is purely formal or has some physical interpretation, we do not know.

A set of vector fields that commute with the generators of supersymmetry transformations is given by

$$
\varepsilon^{*}=\varepsilon^{A}\left(\partial_{A}-\gamma_{A B}^{\lambda} S^{B} \partial_{\lambda}\right) \text {. }
$$

It is easy to see that:

$$
\left[\varepsilon^{*}, \varepsilon\right]=0 \text {. }
$$

This operator can be used to put supersymmetric invariant constraints on $A(U)$ and to write equations of motion that transform into each other under supersymmetry transformations. As the use of this operator follows exactly what is done in superspace, we refer the reader to the discussion of Salam and Strathdee [10].

The similarity between our formulation of global supersymmetry, and the older superspace formulation suggests that we make a general comparison of superspace with graded manifold theory. We begin by briefly describing the superspace concept and pointing out the crucial differences between superspace and a graded manifold.

Superspace is supposed to be a kind of generalization of a differential manifold on which some of the coordinates are ordinary functions and some of the coordinates are elements of a Grassman algebra. In particular, the standard example has four ordinary coordinate functions $x^{\mu}$ and four anticommuting spinor coordinates $\theta^{A}$. The basic idea is that if one considers any function on superspace $f\left(x^{\mu}, \theta^{A}\right)$ the nilpotence of the $\theta^{A}$ limits the dependence of $f$ on $\theta^{A}$ to the first few terms of a power series. Thus any function of $x^{\mu}$ and $\theta^{A}$ can be expanded in terms of functions of $x^{\mu}$ only to show explicitly the $\theta^{A}$ dependence,

$$
f\left(x^{\mu}, \theta^{A}\right)=f_{0}(x)+f_{A}(x) \theta^{A}+\ldots+f^{4}(x) \theta^{1} \theta^{2} \theta^{3} \theta^{4} .
$$

Such a field on superspace is called a superfield.

Equation (3.14) is analogous to the decomposition of an element of $A(U)$ into the odd generators $S^{A}$ and $C^{\infty}$ functions and a decomposition of (3.14) into irreducible Lorentz covariant fields shows the same particle content as Eq. (2.19). However, in the superspace case the coefficient functions of odd terms are taken to be odd Grassman variables rather than ordinary functions. This difference reflects the different philosophies about classical Fermi fields and is not necessarily serious.

The crucial difference between superspace and graded manifold theory is the following. In superspace one speaks of making translations in the odd dimensions. That is one makes a transformation

$$
\theta^{A} \rightarrow \theta^{A}+\varepsilon^{A},
$$

where the components of $\varepsilon^{A}$ anticommute with themselves and with the $\theta^{A}$. This is supposed to be analogous to a translation in ordinary spacetime

$$
x^{\mu} \rightarrow x^{\mu}+a^{\mu}
$$


and therefore the $\varepsilon^{A}$ are taken to be algebraically independent of the $\theta^{A}$. The possibility of such translations gives meaning to the idea that the $\theta^{A}$ represent new dimensions of the spacetime manifold. Such translations are impossible in graded manifold theory because by definition any odd element must be made up of an odd number of $S^{A}$ s and is thus not algebraically independent of them.

What are the consequences of this difference for the comparison between superspace and graded manifold theory?

In superspace translations such as Eq. (3.10) generate supersymmetry transformations. These transformations are interpreted as passive coordinate transformations and objects defined on superspace are supposed to be invariant under supersymmetry transformations for the same reason normal geometric objects are invariant under general coordinate transformations. This is in contrast to the active interpretation of supersymmetry transformations that we have found in graded manifold theory.

Moreover superspace is sometimes postulated to possess an extended general coordinate invariance in which the ordinary and nilpotent coordinates are transformed into arbitrary functions of each other [12]. This implies that the symmetry group of the tangent space is OSP $(4 ; 3,1)$ which mixes up the even and odd vector fields, rather than just $0(3,1)$ as in our case. As a result of this very large gauge symmetry group, most of the fields represented in the superspace formalism turn out to be gauge artifacts. Since we have formulated supersymmetry in terms of active rather than passive transformations, we do not have such a gauge invariance and thus cannot transform away various component fields of $A(U)$. On the other hand, since they are not mixed up by a passive symmetry group the individual component fields are individually well defined geometric objects and can be projected out at will. In particular we can always project out the normal $C^{\infty}$ part which has the same effect as the superspace gauge in which $\theta^{A}$ is transformed to 0 .

In the superspace formalism one defines invariant actions by integrating some superfield over both commuting and anticommuting variables. The integration theory for Grassman variables is defined by the condition that

$$
\int d \theta^{A} f\left(\theta^{A}+\varepsilon^{A}\right)=\int d \theta^{A} f\left(\theta^{A}\right)
$$

and thus depends on the possibility of translation in the odd coordinates. In graded manifold theory condition (3.17) does not make sense, and it is therefore not clear that the integration theory for graded manifolds should be the same as in superspace. The construction of an integration theory for graded manifolds is a non-trivial mathematical problem on which there has been progress only recently [11]. Unfortunately, until we have an integration theory we do not have a canonical procedure for constructing invariant actions on graded manifolds.

It is clear from our discussion about Eq. (3.15) that graded manifold theory does not provide a formulation for superspace. Or, if it did, it would have to be in terms of an infinite dimensional exterior algebra to allow for arbitrary, algebraically independent translation in the odd coordinate. On the other hand, it is also clear from our discussion that most probably any superspace construction that does not depend on the existence of such odd translations, such as the original 
formulation of global supersymmetry, can be rewritten in terms of graded manifold theory.

Moreover, recent progress in constructing a superspace formulation of supergravity $[13,14]$ makes use of a restricted superspace invariant under a restricted group of symmetry transformations; in particular, their tangent space group is only the Lorentz group. These superspaces are isomorphic to graded manifolds (apart from the problem of the commutivity properties of the coefficient functions), and the supergravity constructions can be rewritten in the terms of this paper [15].

In general, the differences between superspace and graded manifold theory arise from the two different ways the formulations are intended as an extension of spacetime structure. In superspace the spacetime manifold itself is extended and graded, and then the ordinary spacetime structures are simply applied to this bigger space. In graded manifold theory the spacetime itself is not touched, and it is the algebra of functions on spacetime that is extended.

Thus, while superspace and graded manifold theory are in their general formulations incompatible, after the restrictions are made that have been found in each case necessary to successfully formulate supersymmetric theories, they are essentially equivalent. These restrictions are to a spin-graded manifold, in the case of graded manifold theory, and the limitation of the tangent space group to the Lorentz group, in the case of superspace.

\section{Local Supersymmetry and Supergravity}

At the end of Sect. II we define vector fields on a graded manifold and argued that, acting on elements of $A(U)$, they should generate supersymmetry transformations. In Sect. III we showed how constant odd vector fields generated the standard global supersymmetry transformations and that the graded Lie algebra of the constant vector fields gave the usual global supersymmetry algebra. In this section we pass to the case of local supersymmetry by letting the parameters $\varepsilon$ depend on spacetime. We show how supersymmetry transformations act on geometric objects other than functions by generalizing the notion of a Lie derivative to graded manifold theory. We then define a metric structure for graded manifolds and show how the supersymmetry transformations in supergravity can be understood as the Lie derivative, by an odd vector field, of this extended metric.

An ordinary vector field $v$ defines a family of curves, or flow lines, on the manifold to which the vector field is everywhere tangent. One can define a one parameter group of diffeomorphisms of the manifold $\phi_{t}: M \rightarrow M$ by mapping a given point to a point some parameter distance $t$ along the flow line passing through it. This in turn induces an active transformation of the geometric objects on the manifold which is called the Lie transport of the object along the vector field.

The Lie derivative of a geometric object along a vector field is then defined by the rate of change of the object as it is dragged along the vector field [5]. For an ordinary function one can show that the Lie derivative is just given by the action of the vector field

$$
\mathscr{L}_{v} f=v(f)
$$


and that the Lie derivative of one vector field $w$ by another $v$ is given by the commutator

$$
\mathscr{L}_{v} w=[v, w],
$$

where the commutator of two vector fields is defined by

$$
[v, w] f=v(w(f))-w(v(f))=-[w, v] f .
$$

Now the odd vector fields of a graded manifold are not tangent to any curves on the manifold and hence do not generate one parameter groups of diffeomorphisms. However, we can define the Lie derivative by a general vector field on a graded manifold directly by Eqs. (4.1) and (4.2). We must however be careful to replace the commutator by the graded commutator

$$
[v, w] f=v(w(f))-(-1)^{0(v) 0(w)} w(v(f)) .
$$

The graded Lie derivative acting on one forms can be defined by,

$$
\left\langle\mathscr{L}_{v} \phi, w\right\rangle=-(-1)^{0(v) 0(\phi)}\left\langle\phi, \mathscr{L}_{v} w\right\rangle+\mathscr{L}_{v}\langle\phi, w\rangle .
$$

The graded Lie derivative of a higher tensorial object is then defined by the graded Liebnitz rule,

$$
\mathscr{L}_{v}(x \otimes y)=\left(\mathscr{L}_{v} x\right) \otimes y+(-1)^{0(v) 0(x)} x \otimes\left(\mathscr{L}_{v} y\right) .
$$

The graded Lie derivative defined in this way can be seen to satisfy all of the properties that normal Lie derivatives satisfy, including the very important relation

$$
\left[\mathscr{L}_{v}, \mathscr{L}_{w}\right]=\mathscr{L}_{[v, w]} .
$$

This equation tells us that if we define supersymmetry transformations in terms of the graded Lie derivative, the supersymmetry transformations will always satisfy a closed commutator algebra.

We thus shall consider local supersymmetry transformations generated by vector fields of the form (3.6) where we now consider the parameters $\varepsilon^{A}$ to be spacetime dependent $C^{\infty}$ functions.

As a result of the spacetime dependence of the $\varepsilon^{A}$, the commutator of two such vector fields has additional terms and Eq. (3.7) becomes,

$$
\begin{aligned}
{\left[\varepsilon_{1}, \varepsilon_{2}\right]=} & 2 \varepsilon_{1}^{A} \gamma_{A B}^{\lambda} \varepsilon_{2}^{B} \partial_{\lambda}+\varepsilon_{2}^{C} \gamma_{C D}^{\varrho} S^{D}\left(\partial_{\varrho} \varepsilon_{1}^{A}\right)\left(\partial_{A}+\gamma_{A B}^{\lambda} S^{B} \partial_{\lambda}\right) \\
& +\varepsilon_{1}^{C} \gamma_{C D}^{\lambda} S^{D}\left(\partial_{\lambda} \varepsilon_{2}^{A}\right)\left(\partial_{A}+\gamma_{A B}^{\lambda} S^{B} \partial_{\lambda}\right) .
\end{aligned}
$$

The existence of these new terms should not be surprising. In a sense the constant term in the commutator given by Eq. (3.7) can be considered as arising from the intrinsic local structure of the graded manifold in the same sense as the commutators of the constant vector fields on a Lie group give the associated Lie algebra. The new terms are just a consequence of the spacetime dependence of the vector fields as in the case of the commutator of ordinary vector fields on spacetime. Also, note that the extra terms all contain explicit $S^{A}$ 's and hence can be gotten rid of at any time by a projection using the $\sim$ map. 
We now come to the definition of a metric structure for graded manifolds. The most general definition we can make is that the metric is a smooth bilinear map,

$h: T_{p} \otimes T_{p} \rightarrow \operatorname{Ext} V$.

It follows that given a basis $d x^{\mu}, d S^{A}$ for the cotangent space $h$ can be expanded out in components

$$
\begin{aligned}
h= & h_{\alpha \beta} d x^{\alpha} \otimes d x^{\beta}+\phi_{\mu A} d x^{\mu} \otimes d S^{A} \\
& +\phi_{A \mu} d S^{A} \otimes d x^{\mu}+h_{A B} d S^{A} \otimes d S^{B},
\end{aligned}
$$

where $h_{\alpha \beta}, \phi_{\mu A}, \phi_{A \mu}$ and $h_{A B} \in A(U)$, with $h_{\alpha \beta}=h_{\beta \alpha}, h_{A B}=-h_{B A}$.

In order that this extended metric measure ordinary distances on the manifold correctly, we have to require that $h$ acting on two ordinary vector fields gives the ordinary metric on the manifold. This requirement fixes the components

$$
h_{\mu \nu}=g_{\mu \nu},
$$

where $g_{\mu \nu}$ is the ordinary metric on the manifold. Similarly, the graded metric acting on two ordinary spinor fields of the form $\chi=\chi^{A} \partial_{A}$ should play the role of the charge conjugation matrix and this fixes

$$
h_{A B}=C_{A B} \text {. }
$$

Only the $\phi_{A_{\mu}}$ and the $\phi_{\mu A}$ are left unrestricted by such a principle of correspondence. These components allow us to define an inner product between vector and spinor fields, i.e.

$$
h\left(\partial_{\mu}, \partial_{A}\right)=\phi_{\mu A} .
$$

For simplicity we shall take

$$
\phi_{\mu A}=\phi_{A \mu} \text {. }
$$

The contravariant metric tensor can be defined acting on the cotangent space,

$$
h^{*}: T_{p}^{*} \otimes T_{p}^{*} \rightarrow \operatorname{Ext} V
$$

and can be written out as

$$
\begin{aligned}
h^{*}= & h^{* \alpha \beta} \partial_{\alpha} \otimes \partial_{\beta}+\psi^{\alpha A} \partial_{\alpha} \otimes \partial_{A} \\
& +\psi^{A \alpha} \partial_{A} \otimes \partial_{\alpha}+h^{* A B} \partial_{A} \otimes \partial_{B} .
\end{aligned}
$$

The components of the covariant and contravariant metric tensors are related by

$$
\begin{gathered}
h^{* \alpha \beta} g_{\beta \gamma}+\psi^{\alpha A} \phi_{A \gamma}=\delta_{\gamma}^{\alpha}, \\
\psi^{\alpha C} C_{C A}+h^{* \alpha \beta} \phi_{\beta A}=0, \\
h^{* A B} \phi_{B \mu}+\psi^{A \varrho} g_{\varrho \mu}=0, \\
h^{* A B} C_{B C}+\psi^{A \mu} \phi_{\mu C}=\delta_{C}^{A} .
\end{gathered}
$$


We can solve these equations if we set

$$
\begin{aligned}
& h^{* \mu \nu}=g^{\mu \nu}+\varrho^{\mu \nu}, \\
& h^{* A B}=C^{A B}+\tau^{A B},
\end{aligned}
$$

where $g^{\mu v} g_{v \sigma}=\delta_{\sigma}^{\mu}$ and $C^{A B} C_{B C}=\delta_{C}^{A}$ as usual. We find that,

$$
\begin{aligned}
& \varrho_{v}^{\mu}=\psi^{\mu A} \phi_{A v}, \\
& \tau_{C}^{A}=\psi^{A \mu} \phi_{\mu C},
\end{aligned}
$$

where $\psi^{\mu A}$ satisfies the equation

$$
\psi^{\mu C}\left(C_{C A}+\phi_{C}^{\varrho} \phi_{\varrho A}\right)=\phi_{A}^{\mu}
$$

which has the formal solution

$$
\psi^{\mu C}=\phi_{A}^{\mu}\left(C^{A C}+\phi^{A \varrho} \phi_{\varrho}^{C}\right)^{-1} .
$$

We thus see that if $\phi_{\mu A}$ is nilpotent, or contains a nilpotent part, only one of $h_{\mu \nu}$ or $h^{* \mu \nu}$ can be purely $C^{\infty}$.

We now are prepared to compute the result of acting with a local supersymmetry transformation on the covariant and contravariant graded metric. We first compute $\mathscr{L}_{\varepsilon}$ acting on the basis vector fields and basis form fields. Using Eqs. (3.6), (4.2), and (4.5), we find that

$$
\begin{aligned}
\mathscr{L}_{\varepsilon} \partial_{\mu} & =-\left(\partial_{\mu} \varepsilon^{A}\right) \partial_{A}-\left(\partial_{\mu} \varepsilon^{A}\right) \gamma_{A B}^{\lambda} S^{B} \partial_{\lambda}, \\
\mathscr{L}_{\varepsilon} \partial_{A} & =\varepsilon^{c} \gamma_{C A}^{\lambda} \partial_{\lambda}, \\
\mathscr{L}_{\varepsilon} d x^{\nu} & =\left(\partial_{\lambda} \varepsilon^{A}\right) \gamma_{A B}^{\nu} S^{B} d x^{\lambda}+\varepsilon^{C} \gamma_{C A}^{\nu} d S^{A}, \\
\mathscr{L}_{\varepsilon} d S^{A} & =-\left(\partial_{\mu} \varepsilon^{A}\right) d x^{\mu} .
\end{aligned}
$$

We see that vector fields and one form fields have rather different supersymmetry transformation rules. This is because the Lie derivative satisfies Eq. (4.5); form fields transform so as to compensate the transformation of vector fields. One consequence of this is that the components of the contravariant metric tensor will necessarily have a different behavior under supersymmetry transformations than the components of the covariant metric tensor.

We calculate first the effect of $\mathscr{L}_{\varepsilon}$ on the contravariant metric tensor. Using Eq. (4.16) and the graded Liebnitz rule we find that,

$$
\begin{aligned}
\mathscr{L}_{\varepsilon} h= & -\left(\partial_{\mu} \varepsilon^{A}\right) h^{* \mu \nu}\left(\partial_{\nu} \otimes \partial_{A}+\partial_{A} \otimes \partial_{\nu}\right) \\
& +\varepsilon^{B} \gamma_{B A}^{\mu} h^{* A C} \partial_{\mu} \otimes \partial_{C}-\varepsilon^{D} \gamma_{D B}^{\mu} h^{* A B} \partial_{A} \otimes \partial_{\mu} \\
& +\varepsilon^{B} \gamma_{B A}^{\mu} \psi^{A \nu}\left(\partial_{\nu} \otimes \partial_{\mu}\right)-\psi^{\mu A}\left(\partial_{\mu} \varepsilon^{B}\right)\left(\partial_{B} \otimes \partial_{A}-\partial_{A} \otimes \partial_{B}\right) \\
& -\left(\partial_{\nu} \varepsilon^{A}\right) \gamma_{A B}^{\varrho} S^{B} h^{* \mu \nu}\left(\partial_{\mu} \otimes \partial_{\varrho}+\partial_{\varrho} \otimes \partial_{\mu}\right) \\
& -\psi^{\mu A}\left(\partial_{\mu} \varepsilon^{D}\right) \gamma_{D B}^{\varrho} S^{B}\left(\partial_{A} \otimes \partial_{\varrho}+\partial_{\varrho} \otimes \partial_{A}\right) \\
& +\varepsilon^{A} \gamma_{A B}^{\lambda} S^{B}\left(\partial_{\lambda} h^{* \mu \nu}\right) \partial_{\mu} \otimes \partial_{v} \\
& +\varepsilon^{D} \gamma_{D E}^{\lambda} S^{E}\left(\partial_{\lambda} h^{* A B}\right) \partial_{A} \otimes \partial_{B}+\varepsilon^{A}\left(\partial_{A} h^{* \mu \nu}\right) \partial_{\mu} \otimes \partial_{v} \\
& +\varepsilon^{A}\left(\partial_{A} \psi^{B \mu}\right) \partial_{B} \otimes \partial_{\mu}+\varepsilon^{A}\left(\partial_{A} h^{* B C}\right) \partial_{B} \otimes \partial_{C} .
\end{aligned}
$$


We interpret this transformation as a transformation of the different coefficient functions of Eq. (4.11), as we did with global supersymmetry in Sect. III. We find that,

$$
\begin{aligned}
\delta h^{* \mu \nu}= & \varepsilon^{B} \gamma_{B A}^{(\mu} \psi^{v) A}-g^{\lambda(\mu}\left(\partial_{\lambda} \varepsilon^{A}\right) \gamma_{A B}^{\nu)} S^{B} \\
& +\varepsilon^{A} \gamma_{A B}^{\lambda} S^{B} \partial_{\lambda} h^{* \mu \nu} \\
& +\varepsilon^{A} \partial_{A} h^{* \mu \nu} \\
\delta \psi^{\mu A}= & -h^{* \mu \nu}\left(\partial_{\nu} \varepsilon^{A}\right)-h^{* A B} \gamma_{B D^{\mu}}^{\mu} \varepsilon^{D} \\
& -\psi^{\lambda A}\left(\partial_{\lambda} \varepsilon^{D}\right) \gamma_{D B}^{\mu} S^{B} \\
& +\varepsilon^{B} \partial_{B} \psi^{\mu A} \\
\delta h^{* A B}= & -\psi^{\mu[A} \partial_{\mu} \varepsilon^{B]}+\varepsilon^{D} \gamma_{D E}^{\lambda} S^{E} \partial_{\lambda} h^{* A B} \\
& +\varepsilon^{A} \partial_{A} h^{* A B}
\end{aligned}
$$

Equations (4.18a) and (4.18b) are the usual supergravity transformation laws with $h^{* \mu v}$ in the place of $g^{\mu v}$ and plus some additional terms. These additional terms contain explicit $S^{A}$ 's of $\partial_{A}$ 's and depend only on the components of whose variation they are a part. Thus they represent the mixing of the $C^{\infty}$ parts with the nilpotent parts of the individual components. If the component fields are purely $C^{\infty}$ then the $\partial_{A}$ terms are absent, and the terms with $S^{A}$ can be eliminated by a projection.

The remaining $\delta \psi$ variation is of the form $\delta \psi \approx\left(\partial^{\mu}+h^{* A B} \gamma_{B D}^{\mu}\right) \varepsilon^{D}$. Terms containing $\gamma^{\mu}$ occur in the $\delta \psi$ variation in the recently discovered transformation laws for supergravity with auxiliary fields [16]. Another feature this formulation and the auxiliary field formulation share is that the algebra of supersymmetry transformations closes without recourse to the question of motion [in our case this follows from Eqs. (4.7) and (4.8)]. This suggests that the auxiliary fields may result from a decomposition of the $h^{* A B}$ components; indeed the $h^{* A B} \gamma^{\mu} \varepsilon$ term in the variation comes from the mixing in of the $h^{* A B}$ term in the metric. In fact, we can write

$$
h^{* A B}=S C^{A B}+P\left(\gamma^{5}\right)^{A B}+A_{a}\left(\gamma^{5} \gamma^{a}\right)^{A B}
$$

exhibiting explicitly the auxiliary fields. Unfortunately, Eq. (4.18c) does not seem to be of the right form to give all of the variations of the auxiliary fields given in [16].

It is interesting to note the origin of the other terms in the supergravity transformation laws. The $\partial \psi \sim \partial \varepsilon$, comes from

$$
\mathscr{L}_{\varepsilon^{A} \hat{\partial}_{A}} \partial_{\mu}=\left[\varepsilon^{A} \partial_{A}, \partial_{\mu}\right]=-\left(\partial_{\mu} \varepsilon^{A}\right) \partial_{A} .
$$

This is completely analogous to the ordinary Lie derivative of the ordinary metric $\delta g \sim \nabla \xi$ which comes from

$$
\mathscr{L}_{\xi^{\mu} \partial_{\mu}} \partial_{\nu}=\left[\xi^{\mu} \partial_{\mu}, \partial_{\nu}\right]=-\left(\partial_{\nu} \xi^{\mu}\right) \partial_{\mu} .
$$


The $\delta g \sim \bar{\varepsilon} \gamma \psi^{\mu}$ term, on the other hand, has its origin in the raising term $\varepsilon^{A} \gamma_{A B}^{\mu} S^{B} \partial_{\mu}$ which acts like

$$
\left[\varepsilon_{A B}^{A \gamma^{\mu}} S^{B} \partial_{\mu}, \partial_{C}\right]=\varepsilon^{A} \gamma_{A C}^{\mu} \partial_{\mu} .
$$

We see from this that the $\delta g=\bar{\varepsilon} \gamma \psi$ variation in supergravity arises from exactly the same operation that generates the $\delta \chi=\not \partial \phi \varepsilon$ term in the scalar multiplet of global supersymmetry.

We have already remarked that the supersymmetry transformations of the covariant and of the contravariant metric tensors must be different. This means that we will find different transformation laws for supergravity depending on whether we consider the $g_{\mu \nu}$ and $\psi_{\mu A}$ field to be part of the covariant or contravariant graded metric tensor. This is a consequence neither of our active interpretation of supersymmetry transformations, nor of any detail of our particular formulation, but only of our assumption that supergravity is to be constructed by putting the $g_{\mu \nu}$ and $\psi_{\mu A}$ fields together into some kind of a generalized metric tensor. The same thing must be true in superspace or in any other attempt at a geometric formulation that makes this assumption.

Following the same procedure as before, we find the supersymmetry transformations of the components of the covariant graded metric tensor to be,

$$
\begin{aligned}
\delta h_{\mu \nu}= & \left(-\phi_{A(\mu}+S^{B} \gamma_{(\mu B A}\right) \partial_{\nu)} \varepsilon^{A}+\left(\mathscr{L}_{\varepsilon} h_{\mu \nu}\right), \\
\delta \phi_{\mu}^{A}= & \partial_{\mu} \varepsilon^{A}+\gamma_{\mu B}^{A} \varepsilon^{B}+\phi_{\lambda}^{A}\left(\partial_{\mu} \varepsilon^{D}\right) \gamma_{D E}^{\lambda} S^{E} \\
& +\left(\mathscr{L}_{\varepsilon} \phi_{\mu B}\right) C^{A B} . \\
\delta h_{A B}= & \phi_{A \mu} \gamma_{B C}^{\mu} \varepsilon^{C}+\left(\mathscr{L}_{\varepsilon} h_{A B}\right),
\end{aligned}
$$

where we have used Eqs. (4.6), (4.9), (4.10), and (4.16) and we have not calculated out the variations of the component fields themselves.

We see that the $\delta \phi$ term is of essentially the same form as the $\delta \psi$ term, while the $\delta h_{\mu \nu}$ and $\partial h_{A B}$ terms have, in a sense, the opposite dependence as in the contravariant case. That is $\delta h_{\mu \nu} \sim \phi \partial \varepsilon$, while $\delta h^{* A B} \sim \psi \partial \varepsilon$ and $\delta h_{A B} \sim \bar{\varepsilon} \gamma \phi$ while $\delta h^{* \mu \nu} \sim \bar{\varepsilon} \gamma \psi$.

The results of this section raise several interesting questions. Why is it the transformation of the contravariant components that gives the correct supergravity transformation law for the metric? Might the covariant transformation laws also play some role in supergravity theory?

\section{Conclusion}

In the investigations leading to this paper we had in mind two closely related goals. The first was to develop a conceptual understanding of supergravity theory in terms similar to those used in relativity theory. The second was to develop a geometric formulation for supergravity that was in harmony with the conceptual understanding we sought and that was, at the same time, mathematically respectable. Here we would like to summarize our basic conclusions.

Physical arguments led us to the conclusion that there cannot be a sensible formulation in which supergravity transformations have a passive interpretation, and that therefore the spin $3 / 2$ field cannot be related to a connection structure. 
These points alone rule out many otherwise attractive possibilities for a geometric formulation of supergravity.

We then found that the graded manifold theory of Kostant does provide an adequate base for a geometric formulation of supergravity. The main idea of this formulation is the following: The algebra of functions on spacetime is extended to a graded commutative algebra, which is chosen to be the algebra of sections through the exterior bundle of the spin bundle. Physically, this corresponds to a particular way of extending the algebra of classical observables of a scalar field to include the observables of spinning fields. A straightforward generalization of differential geometry is then made in which the extended algebra plays the role of the function algebra and the resulting formalism is found to contain a representation of supergravity theory.

Indeed this formulation of supergravity theory turns out to be equivalent to the recent superspace supergravity constructions [15]. In spite of this formal equivalence we would like to argue that the spin graded manifold provides a geometrical formulation for supergravity that is closer to the physical content of the theory.

As a result of the fact that the construction is based directly on the spin bundle each component of a graded function is individually defined by its Lorentz transformation properties, and thus has an invariant meaning. As a result of this all of the transformations allowed by the formalism, with the exception of general coordinate transformations and local Lorentz rotations, must be active, as corresponds to our physical reasoning. In the superspace formulations, on the other hand, the physical fields are not defined diretcly in terms of the components in the $\theta$ expansion and one must introduce spinorial covariant derivatives, or more properly frame fields, to pick out the physical fields in terms of their Lorentz transformation properties $[14,15]$. This is related to the fact that in these formulations one has a large amount of freedom to make additional passive transformations. These transformations reflect the idea that superspace is an extension of spacetime with additional dimensions coordinatized by anticommuting numbers, and that therefore arbitrary general coordinate transformations mixing up the $\theta$ 's should be allowed. However these transformations play no role in the physics analogous to the role played by gauge transformations in YangMills theory and are completely unphysical. When these extra degrees of freedom are frozen out, by a procedure known as going to Wess-Zumino gauge, the result is essentially the formulation given in this paper.

Of course many questions still remain to be answered about the application of graded manifold theory to supergravity, and we leave the reader with two of the most important. First, will an investigation of the integration theory for a graded manifold lead to the usual superspace integration formulas? Second, will an extension of graded manifold theory to include internal symmetries lead to a geometrical formulation for extended supergravity?

Acknowledgements. We would like to thank Michael Forger for pointing out to us, and insisting, that Kostant's graded manifold theory is the right mathematics for supersymmetry. We would also like to thank Marjorie Batchelor, Jim Gates, Bertrand Kostant, Jean Thiery-Mieg, Martin Roček, Graeham Segal, and Warren Siegel for discussions that deepened our understanding of the subject of this paper. This paper is dedicated to Herbert Bernstein, who taught us physics. 


\section{References}

1. Golfand, Yu.A., Likhtman, E.P.: JETP Letters 13, 452 (1971)

2. Wess, J., Zumino, B.: Nucl. Phys. B 70, 39 (1974); Phys. Lett. B 49, 52 (1974)

3. Freedman, D.Z., Nieuwenhuizen, P. van, Ferrara, S.: Phys. Rev. D 13, 3214 (1976); Freedman, D.Z., Nieuwenhuizen, P. van : Phys. Rev. D 14, 912 (1976)

4. Deser, S., Zumino, B.: Phys. Lett. B 62, 335 (1976)

5. Trautman, A.: In: Lectures on general relativity; Brandeis 1964, Summer Institute on Theoretical Physics, Vol. I. Englewood Cliffs, NJ : Prentice Hall 1965; Hawking, S.W., Ellis, G.F.R.: The large scale structure of spacetime. New York: Cambridge University Press 1973

6. Kostant, B. : Graded manifolds, graded Lie theory and prequantization. In : Differential geometric methods in mathematical physics. Lecture notes in mathematics, Vol. 570. Berlin, Heidelberg, New York: Springer 1977

7. Noether, E.: Gött. Nachr. 235 (1918)

8. Batchelor, M.: Proc. Am. Math. Soc. (to be published)

9. Volkov, D.V., Akulov, A.D.: Phys. Lett. B46, 109 (1973); Salam, A., Strathdee, J.: Nucl. Phys. B76, 477 (1974); Ferrara, S., Wess, J., Zumino, B.: Phys. Lett. B 51, 239 (1974)

10. Salam, A., Strathdee, J.: Phys. Rev. D 11, 1521 (1975)

11. Batchelor, M.: Personal communication

12. Nath, P., Arnowitt, R.: Phys. Lett. B56, 177 (1975); Nath, P.: Supersymmetry and gauged supersymmetry. In: Gauge theories and modern field theory. Arnowitt, R., Nath, P. (eds.). Cambridge, MA: MIT Press 1976

13. Siegal, W.: Preprints, Harvard University (Dec. 1977); Gates, S.J., Siegal, W.: Preprint, Harvard University (Aug. 1978); Nucl. Phys. B (submitted)

14. Wess, J., Zumino, B.: Phys. Lett. B 74, 51 (1978); Zumino, B.: Cargese Summer School Lectures 1978. New York, London: Plenum Press (to be published)

15. Smolin, L. : Preprint HUTP-78/AO48, Harvard University (Nov. 1978)

16. Stella, K.S., West, P.C.: Phys. Lett. B 74, 330 (1978); Ferrara, S., Nieuwenhuizen, P. van : Phys. Lett. B 74, 333 (1978)

Communicated by R. Geroch

Received November 2, 1978 
